\title{
EFFECT OF ER,CR:YSGG LASER BIO-MODULATION OF OSTEOTOMY SITE ON PERI-IMPLANT CRESTAL BONE LEVEL IN LOWER SINGLE FIXED DETACHABLE PROSTHESIS
}

\author{
Mona M. Aboelnagga *, Hesham Ebrahim Alam Eldeen ${ }^{* *}$ and Amany Khalifa El-Sayed ${ }^{* * *}$
}

\begin{abstract}
Objectives: This research was carried out to evaluate the effect of bio-modulation of the osteotomy site with Er,Cr:YSGG LASER on peri-implant crestal bone level changes in cases having edentulous mandible opposed with maxillary natural teeth rehabilitated with fixed detachable prosthesis constructed from BioHPP (High Performance Polymer) using CAD/CAM technology.
\end{abstract}

Materials and Methods: This is a split mouth design study in which ten male patients exhibiting completely edentulous mandibular arch opposed by dentate maxillary arch were rehabilitated with fixed detachable lower single denture supported by six dental implants constructed from BioHPP. The osteotomy segment bio-modulated using Er,Cr:YSGG $(2790 \mathrm{~nm})$ LASER was randomly selected and considered as the study group while the other segment (non laser mediated osteotomy) as the control group. Lower single complete dentures were constructed to all participants following the same basic principles. All patients were rehabilitated with fixed detachable lower dentures using Computer-aided design/computer-aided manufacturing (CAD/CAM) technology supported by six dental implants. The application of 3 dimensional (3D) imaging (computerized tomography scan), 3D software for proper treatment planning, and fabrication of computer-generated surgical guides using additive prototyping were followed for all patients. Surgical installation of the implants was performed under strict aseptic conditions The selected osteotomy segment (study group) was biomodulated using Er,Cr:YSGG (2790 nm) LASER and the implants were immediately loaded using the modified existing denture. After 3 months, the patients were scheduled for construction of the definitive prosthesis from which milling was performed in BioHPP polyether-ether ketone (PEEK) disc material. Peri-implant crestal bone level changes were assessed using digital periapical radiographs taken with the standardized long cone paralleling technique at six, twelve and eighteen months after loading. Data collected were statistically analyzed using (ANOVA-test $\mathrm{P}<0.05$ ).

\footnotetext{
* Associate Professor of Prosthodontics, Faculty of Dentistry, Ain Shams University, Egypt

** Associate Professor of Prosthodontics, Faculty of Oral and Dental Medicine, Future University, Cairo, Egypt

*** Assistant professor of Oral \& Maxillofacial Surgery, Faculty of Oral and Dental Medicine, Modern University for

Technology and Information.
} 
Results: This study showed that there was a statistically significant increase in crestal bone height loss around the implants in both groups. However, statistically insignificant difference between the two groups was found, where the studied group showed less bone resorption from loading to eighteen months in comparison to the control group.

Conclusion: Within the limitation of this study findings, it seems reasonable to suggest that patient compliance, control of occlusal load, primary implant stability, good oral hygiene and passive fit are important factors for the long term success of fixed detachable lower single denture designed by CAD/CAM rather than the additional use of low level laser therapy.

KEY WORDS: Bio-modulation, LASER, Implant, lower single denture, Fixed detachable prosthesis, BioHPP

\section{INTRODUCTION}

Over the past several years, the use of implant prosthesis is considered the standard line of treatment for the rehabilitation of single denture. This treatment option offers considerable functional advantages for patients rehabilitated with single mandibular denture. ${ }^{(1,2)}$ Despite adequate denture fabrication of lower single denture, it is not possible in many instances to achieve optimum conventional denture and to preserve the remaining residual ridge as well. ${ }^{(3,4)}$

The occlusal form of the remaining natural teeth and the impact of occlusal forces induced by the static dentate maxillary arch during function initiate deleterious effects on the supporting tissues. In addition given the fact that reduction of the mandible is on average four times as greater as reduction of the maxilla makes the construction of mandibular single denture more problematic than maxillary single denture. The situation is often compounded in cases having severe mandibular residual ridge resorption thus making conventional treatment nearly impossible. ${ }^{(3-5)}$

Implant treatment options may vary from the use of removable implant supported overdenture to the construction of fixed implant supported prosthesis. An alternative to fixed prosthesis is the fixed detachable prosthesis (Screw-Retained Dentures). Treatment planning is balanced between the patient's preferences, finances, and clinical factors. ${ }^{(1)}$ The choice of definitive prosthesis for the implant is based on the patients' preference, demands, compliance, manual dexterity and economic status. Moreover, ridge anatomy, maxillomandibular relationship and hygienic maintenance are major factors. ${ }^{(6,7)}$

Oral rehabilitation of edentulous patients with hybrid dentures has been observed to accomplish greater masticatory function and psychological satisfaction than with conventional overdentures. ${ }^{(8-10)}$ Occlusal forces have been increased considerably subsequent to the use of an implant-supported prosthesis. ${ }^{(1)}$ Screw-Retained Dentures provide a fixed implant solution for edentulous patients requiring a stable and esthetic replacement for removable prostheses. Furthermore they are successfully used in combination of tilted and axially positioned implants in the posterior part or resorbed dental arch. In addition, complete denture that is borne totally by implants installed in the interforaminal area results in considerable delay in the resorption process of the posterior mandibular ridge and may even contribute to increase in the amount of posterior bone height even when no posterior implants are inserted. ${ }^{(6,7)}$ Compared to fixed metal ceramic restorations, fixed hybrid prosthesis might be a more reliable treatment option that maintains adequate lip support, phonetics and esthetics. Biomechanical problems associated with inconvenient labial moment arm forces can consequently be avoided. ${ }^{(11)}$ 
Nevertheless, a series of mechanical and infectious-inflammatory complications had been registered with the employment of this type of prosthesis. A passive fit is critical to the long-term success and not achieving it leads to mechanical and biological failures such as peri-implant bone loss, screws loosening or fracture of the abutment or the implant were reported. Although a number of techniques to prevent or adjust distortions that occur during impression making, cast pouring, waxing, casting, indexing, and soldering, inaccuracies in the fit of frameworks persist. ${ }^{(1,12-14)}$ A $50 \mu$ misfit may require the implant to move within the bone 200 $\mu \mathrm{m}$ before the casting fits passively. In addition, loading of the cantilever on the prosthesis can cause a hinging effect that induces considerable stresses on the implant closest to the load application. When the distal cantilevers of full fixed prostheses are extreme in length deformation of the framework can result in fracture of the prosthetic screw, the acrylic resin teeth or even the framework itself. ${ }^{(15,16)}$

The fixed detachable prosthesis can be made on a variable number of implants, with a minimum of four, although it is preferred that should place the biggest number of implants that is possible. However, in the completely edentulous mandible problems such as minimum bone volume, poor bone quality, and the demand for bone-grafting procedures preceding to implant placement create some challenging circumstances. ${ }^{(17)}$

Various material combinations including metal/ acrylic (with metal frame work and resin), multiunit ceramo-metal restorations, CAD/CAM-based restorations with metal, zirconia or BioHPP (High Performance Polymer) frameworks, or monolithic zirconia have been used for constructing fixed detachable prosthesis. ${ }^{(18,19)}$

Loss of the acrylic teeth, lack of natural color primarily in the prosthetic gingival area, plaque accumulation and wear of acrylic are drawbacks of fixed detachable dental prostheses made of metal/ acrylic. In spite of the excellent esthetic effects of prostheses made of metal/porcelain, the major drawback of metal/porcelain prostheses is porcelain chipping. In zirconia/ceramic prostheses, ceramic chipping or breakage of the zirconia framework are the main disadvantages. ${ }^{(20)}$

Polyether ether ketone (PEEK) is a highperformance polymer that can be used as a metal substitute for fixed and removable prostheses. It has several advantages, such as favorable strength-to weight ratio, biocompatibility, corrosion resistance, radiolucency during imaging, low plaque affinity, and chemical stability. ${ }^{21,22)}$ In addition, it has good mechanical performance, such as minimal creep, wear resistance, reduced specific weight, and shock absorption. ${ }^{(21-24)}$ PEEK can be constructed by CAD/ CAM or injection molding. ${ }^{(24)}$ PEEK material was modified by adding 20\% ceramic fillers (BioHPP, high-performance polymer) to increase the modulus of elasticity. BioHPP is as elastic as bone, acts as a stress breaker, and decreases the occlusal forces transmitted to the restoration and opposing dentition. ${ }^{(25)}$

The longevity of dental implants is highly reliant on the integration between implant components and oral tissues, comprising hard and soft tissues. The use of low-level LASER (LLLT) has been proposed as an approach of accelerating and improving the bone tissue healing process. LASER light irradiation has been used in the medical field and showed biostimulatory effects on wound healing, fibroblast proliferation and collagen synthesis. ${ }^{(26)}$ Moreover, LLLT shows an increase in mitochondrial respiration and adenosine triphosphate (ATP) synthesis. Bone irradiated mainly with infrared wavelengths shows improved osteoblastic proliferation, collagen deposition, and bone neoformation when compared to non-irradiated bone. ${ }^{(27-29)}$ Low level laser application stimulates bone formation and maturation around the implants. Studies showed that alveolar bone height was preserved while the 
bone density was increased in the side exposed to low level laser application and they are antibacterial in nature and can be used to varying degrees to disinfect the osteotomy that results in improve dental implant bone contact. ${ }^{(30,31)}$

The erbium family acts predominantly on the surface. It has an absorption depth of approximately $0.01 \mathrm{~mm}$ whereas the $800 \mathrm{~nm}$ diodes can be transferred through the tissue to depths up to $10 \mathrm{~mm}$. ${ }^{(29)}$ Erbium Chromium Doped Yttrium Scandium Gallium Garnet (Er,Cr:YSGG) LASER has a wavelength of $2780 \mathrm{~nm}$, in the infrared spectrum. Its use has been investigated in bone ablation for implant bed preparation. Er,Cr:YSGG LASER is absorbed by water so it allows bone cutting. Advantages of Er,Cr:YSGG include absence of bone dust and vibration, enhanced visualization of the surgical field due to hemostasis and the improved patient comfort with less post operative pain and edema. In addition to ablating bone has a bactericidal effect, Er,Cr:YSGG LASER provides straight, clean and precise bone ablation and causes minimal thermal damage to the adjacent tissues. ${ }^{(32-39)}$

Although the innovation of new materials and CAD/CAM technology for the construction of screw retained based restorations is the point of concern in the clinical studies done in the late years, more detailled information about the effect of low laser therapy on dental implant bone contact in single lower denture is needed. Accordingly, this study was carried out to evaluate the effect of bio-modulation osteotomy site with Er,Cr:YSGG LASER on peri-implant crestal bone level changes in cases of edentulous mandible opposed with dentate maxillary arch rehabilitated with fixed detachable prosthesis constructed from BioHPP (High Performance Polymer).

\section{MATERIALS AND METHOD}

\section{Study design}

This is a split mouth design study in which ten male patients exhibiting completely edentulous mandibular arch opposed by dentate maxillary arch were rehabilitated by fixed detachable lower single denture supported by six dental implants constructed from BioHPP (High Performance Polymer). The selected osteotomy segment (study group) biomodulated using Er,Cr:YSGG (2790 nm) LASER was randomly selected as created in an Excel spread sheet while the other segment (non laser mediated osteotomy) was considered the control group.

The inclusion criteria were adequate bone volume (to accommodate dental implants at least $13 \mathrm{~mm}$ length and $3.5 \mathrm{~mm}$ diameter in the interforaminal and $10 \mathrm{~mm}$ length and $4 \mathrm{~mm}$ in diameter in the first molar area (Interactive Implant, Implant Direct, USA), bone density was verified by preoperative cone beam computed tomography, the residual alveolar ridge covered with firm fibrous mucoperiosteum. Angle's class I ridge relationship, and adequate restorative space at least $12 \mathrm{~mm}$ as indicated by provisional jaw relation. Exclusion criteria were patients with systemic diseases that contraindicate implant surgery as liver disease, any disease of immunity, metabolic and bleeding disorders, osteoporosis, radiation therapy to the head and neck region, immunosuppressive therapy and hyper-parathyroidism; conditions that might affect the oral tissues or the bone metabolic rate. Patients suffering from neuromuscular disorders, temporomandibular joint disorders, with parafunctional habits as bruxism and clenching, smokers were also excluded.

Detailed information about the treatment modality was given to all participants; the surgical and prosthetic steps, the risks and benefits and the treatment alternatives were explained thoroughly. All participants were motivated and were informed 
that they will be a part in a study that requires their co-operation. All patients approved to share and keep on the recommendations and instructions given to them in the form of a signed consent.

\section{Surgical and Prosthetic Interventions}

A provisional centric relation was used to mount maxillary and mandibular casts for setting up of artificial teeth. Necessary modifications and occlusal adjustments were made on the maxillary stone cast. A clear acrylic template was fabricated over the modified stone cast and was used to identify the needed occlusal adjustments intra-orally. The modified natural teeth were polished and stannous fluoride gel was applied topically to the teeth. ${ }^{(3,4)}$

Lower complete dentures were constructed to all participants following the same basic principles. Centric occlusion was established at centric relation. Modified cusped acrylic teeth were used and balanced on semi-adjustable articulator for centric positions. Dentures were clinically remounted to refine the occlusion, to ensure free anterior contact in centric and free non-interfering contact during all excursive mandibular movements. Patients were imformed to wear the dentures for three months to allow for mucosal adaptation. Gutta percha markers were added in a staggered pattern at different levels to the occlusal plane on the denture polished surface. Each patient was double scanned (dual scan protocol). Based on the gutta percha markers,

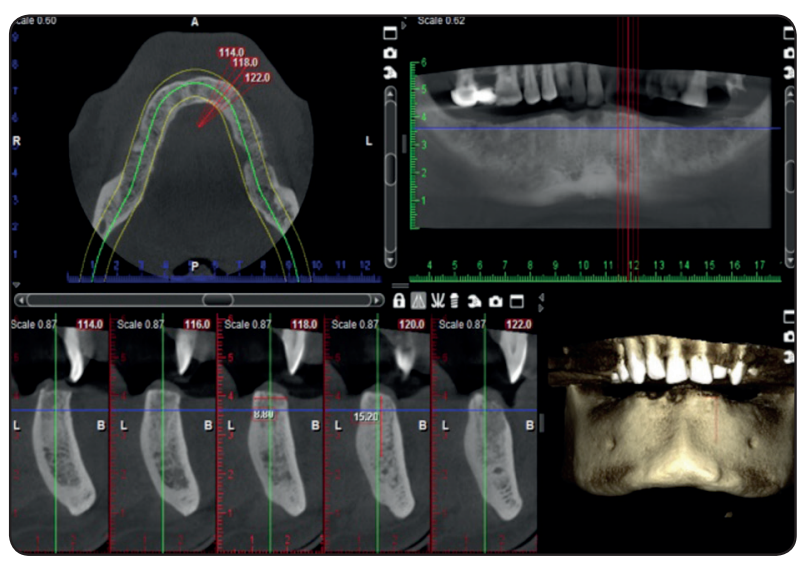

Fig. (1): Cone beam CT evaluation the two scans were superimposed onto each other and the CBCT raw data was converted into 3D information by in 2guide cyber med software where the final file contained reformatted images in 3D bone model, 3D radiological data set and 3D radiographic modified denture guide model. The six implants were virtually designed to simplify the selection of the implants' location, length and diameter according to the patient's bone quantity and approximation to inferior alveolar nerve. (Fig 1,2)

The plan was used to construct a mucosal supported stereolithographic surgical guide with sleeves located over the proposed implant sites. The guide was constructed using rapid prototyping technology. Patients were pre-medicated with 0.2 Cholorohexidine gluconate mouth wash that was prescribed two days before surgery $(2 \mathrm{gm} / \mathrm{d})$ and was continued 7 days after surgery, Amoxicillin Clavulanate Potassium 24 hours before surgery then twice daily for 10 days postoperatively, analgesics were also prescribed. Each patient received six implants using the flapless surgical protocol. At the time of surgery, the surgical guide was stabilized in the patient's mouth by a rubber base interocclusal record and secured to the mandibular bone using the anchor pins. The osteotomy preparation was performed following the report provided with the surgical guide about the exact drill length and diameter to be used according to the virtual implant plan. Fig (3)

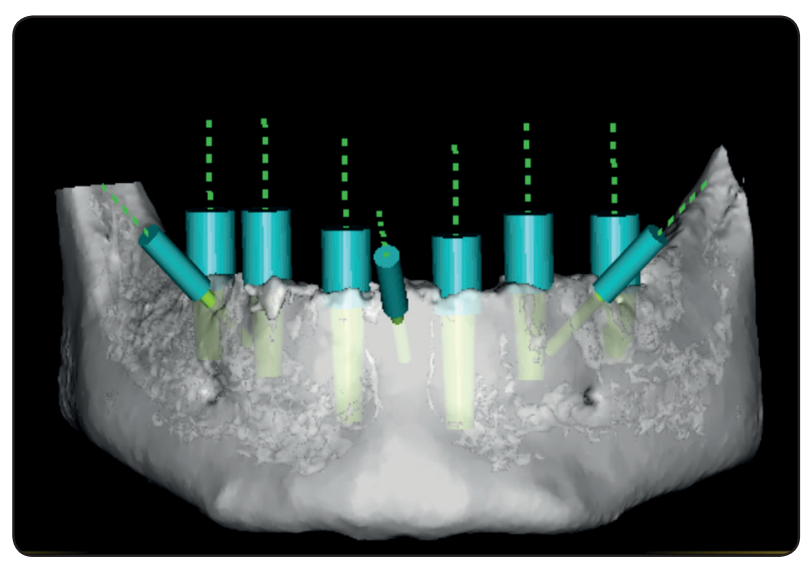

Fig. (2): Implant planning 
The anchorage pins were removed to remove the surgical guide. The selected osteotomy segment (study group) was bio-modulated using Er,Cr:YSGG $(2790 \mathrm{~nm})$ LASER with a radial firing tip RFPT (8 $\mathrm{mm}$ diameter and $14 \mathrm{~mm}$ length) biolase water LASER tip using circular motion from the apex to the marginal bone in a non-contact manner $2 \mathrm{~mm} /$ sec 4 times. Fig (4) The machine was set to 2.5 Watt, $50 \mathrm{~Hz}$ on $\mathrm{H}$ mode. $20 \%$ air and $40 \%$ water was used.

The surgical guide was repositioned and fixed in the exact previous position by the anchorage pins. Implants were inserted through the metal sleeves of the surgical guide, countersinking was avoided to increase implant stability and preserve the marginal bone. Fig (5) For immediate loading protocol, the minimum torque at implant placement was $35 \mathrm{Ncm}$. Then the anchorage pins were removed to remove the surgical guide. Osstell (Osstell ISQ device, Gothenburg, Sweden) was used to evaluate primary implant stability. Average ISQ for immediate loaded implants ranged between 57-60.

Multiunit abutments were screwed to the implants at $20 \mathrm{Ncm}$ and post-operative rotational tomographs were made to check the implant position and passive fit. Temporary titanium caps (titanium sleeves) were screwed to the multiunit abutments and the implants were immediately loaded using the modified existing denture. The denture was modified by removing the labial, buccal and lingual flanges, the denture base opposite to the multiunit abutments was hollowed and the temporary metal caps were picked up in the denture using autopolymerized acrylic resin and the denture was screwed to the implants for immediate loading. Patients were instructed for good oral hygiene. Recall appointments were scheduled to monitor oral hygiene level during the 3 month healing period.

After 3 months, the patients were scheduled for the final impression. Implant verification jig and a custom tray were provided for a direct open tray impression technique using polyether impression material (Pentamix, 3M ESPE). The implant analogues were screwed to the transfer coping. The impression was poured to obtain final stone cast. Implant scan body was attached to the implant analogue for obtaining scannable abutments where scanning was done with desktop scanner. The acquired images were then superimposed on the previous scans of the mandibular single denture and replaced on the computer software by Titanium bases and by superimposing the images, screw positions and pathways were planned in the prosthesis. Cut back for denture teeth and gingiva was done to allow space for denture teeth veneers and gingiva.

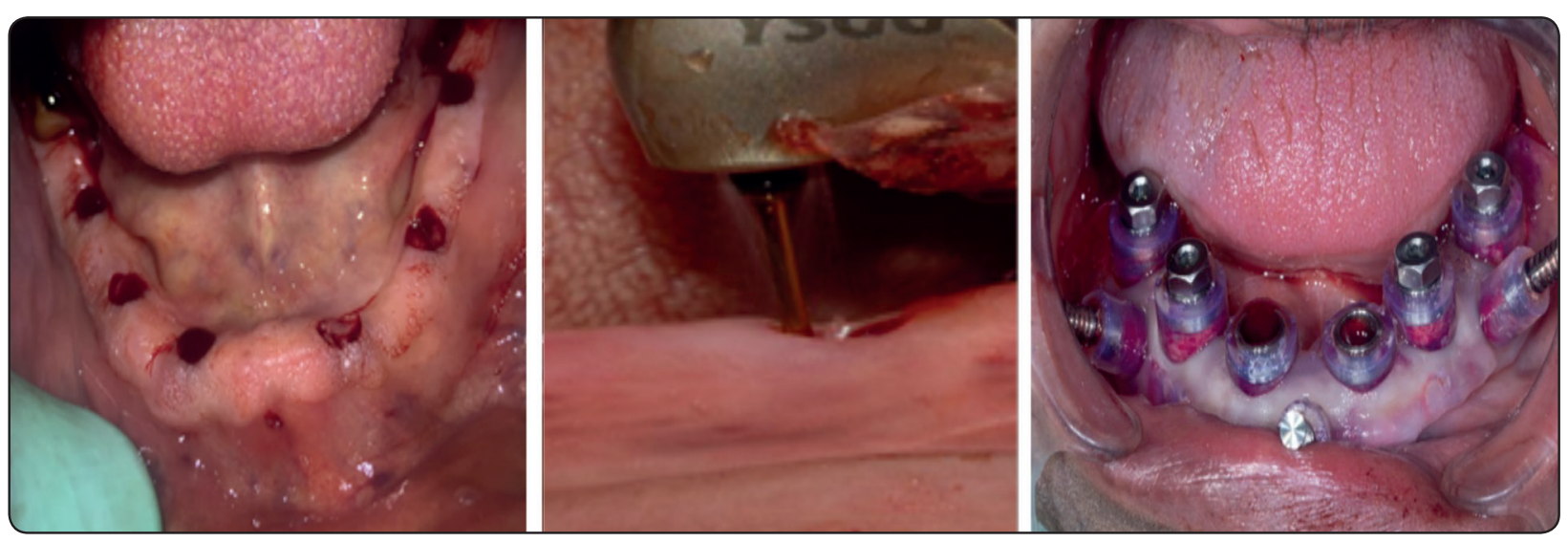

Fig. (3): osteotomy preparation Fig. (4): bio-modulation using Er,Cr:YSGG (2790nm) LASER Fig. (5): Implants were inserted through the metal sleeves of the surgical guide 
The final designed restoration was exported as STL file, from which milling was performed in polyether-ether ketone (PEEK) disc material. BioHPP framework was tried in the patient's mouth for passive fit that was checked visually using a probe and peri-apical radiographs was taken to check for any misfits. It was then confirmed by the one screw test which involved screwing the terminal abutment on one side and checking the fit on the other terminal abutment. The BioHPP framework was painted with suitable adhesive (visio.link, Bredent) for subsequent veneering. Denture teeth veneers (Novolign, Bredent, senden, Germany) (novo-lign A44 for anterior teeth and novo-lign G3P for posterior teeth) were used with the aid of silicone index. Fig (6)

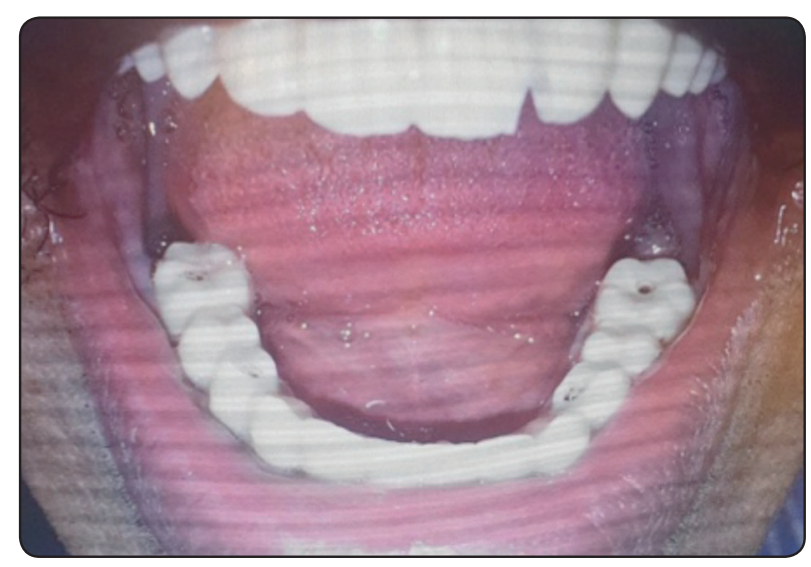

Fig. (6): The final fixed detachable BioHPP restoration

Patients were frequently recalled for inspection, post insertion adjustments, hygiene maintenance and for radiographic assessment of peri-implant marginal bone loss at the time of implant loading, six, twelve and eighteen months. Peri-implant crestal bone level changes were assessed using digital periapical radiographs taken with the standardized long cone paralleling technique using custom made acrylic template and the Rinn-xcp system. ${ }^{(39)}$ The distance between theimplantplatformandthe marginal bone on the mesial and distal sides was measured and averaged for the implants. Implant marginal bone loss was calculated by comparing the bone level at follow up visits to that at baseline.

\section{Statistical analysis}

Numerical data were explored for normality by inspecting the data distribution, calculating the mean and using Kolmogorov-Smirnov and Shapiro-Wilk tests. Data revealed parametric distribution thus it was represented by mean and standard deviation (SD) values. Intra and intergroup comparisons were done utilizing one way analysis of variance (ANOVA test) to test peri-implant crestal bone level changes within the group during different recall appointments and between the two studied treatment modalities followed by Bonferroni test for pairwise comparison. The significance level was set at $\mathrm{P} \leq 0.05$ for all tests. Statistical analysis was performed with IBM ${ }^{\circledR}$ SPSS ${ }^{\circledR}$ (SPSS Inc., IBM Corporation, NY, USA) Statistics Version 25 for Windows.

\section{RESULTS}

All patients attended the follow up recall visits. Patients expressed satisfaction as regard function, retention and stability of their dentures. Clinically, no pain was elicited with palpation or percussion, no exudates were observed in relation to the implants. The study results mean value of peri-implant crestal bone level changes $(\mathrm{mm})$ are represented in table (1) and fig (7)

Statistical analysis of bone height for the studied group (Er,Cr:YSGG LASER $2780 \mathrm{~nm}$ biomodulation) using ANOVA test for repeated measures revealed significant difference in periimplant crestal bone level changes $(\mathrm{mm})$ during the follow intervals $(P \leq 0.05)$. Simple main effect with Bonferrioni correction revealed significant difference between peri-implant crestal bone level changes (mm) from loading - 6 months, 12 months and 18 months and significance difference between bone heights at the interval 6-12 months. However minimal insignificant change $(P \geq 0.05)$ in the measured peri-implant crestal bone level changes (mm) was detected at the interval 12-18 months. 
TABLE (1): The mean and standard deviation $t$ values of peri-implant crestal bone level changes for studied group (Er,Cr:YSGG LASER $2780 \mathrm{~nm}$ biomodulation) and control group during the follow up periods.

\begin{tabular}{cccccccc}
\hline & \multicolumn{2}{c}{$\begin{array}{c}\text { Studied group } \\
\text { (Er,Cr:YSGG LASER biomodulated osteotomy) }\end{array}$} & \multicolumn{2}{c}{$\begin{array}{c}\text { Control group } \\
\text { (non laser mediated osteotomy) }\end{array}$} \\
\hline Interval & Mean \pm S.D & $\mathrm{dt1}$ & $\mathrm{dt} 2$ & $\mathrm{Mean} \pm$ S.D & $\mathrm{dt1}$ & $\mathrm{dt2}$ \\
\hline 0-6 months & $0.342 \pm 0.12$ & $\mathrm{a}$ & $\mathrm{a}$ & $0.381 \pm 0.11$ & $\mathrm{a}$ & $\mathrm{a}$ \\
\hline 6-12 months & $0.391 \pm 0.09$ & $\mathrm{~b}$ & $\mathrm{a}$ & $0.413 \pm 0.072$ & $\mathrm{~b}$ & $\mathrm{a}$ \\
\hline 12-18 months & $0.13 \pm 0.03$ & $\mathrm{~b}$ & $\mathrm{a}$ & $0.16 \pm 0.05$ & $\mathrm{~b}$ & $\mathrm{a}$ \\
\hline $0-12$ months & $0.732 \pm 0.14$ & $\mathrm{c}$ & $\mathrm{a}$ & $0.794 \pm 0.24$ & $\mathrm{c}$ & $\mathrm{a}$ \\
\hline $0-18$ months & $0.862 \pm 0.39$ & $\mathrm{c}$ & $\mathrm{a}$ & $0.954 \pm 0.28$ & $\mathrm{c}$ & $\mathrm{a}$ \\
\hline
\end{tabular}

ANOVA for repeated measures, similar superscript letters indicate non significant difference

dt1 within the group dt2 between the groups

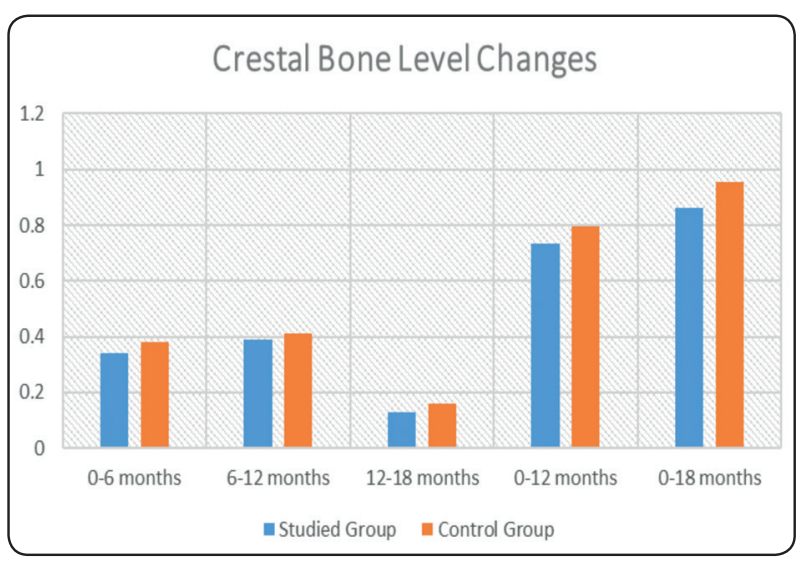

Fig. (7): The mean value of peri-implant crestal bone level changes (mm) for studied group (Er,Cr:YSGG LASER $2780 \mathrm{~nm}$ biomodulation) and control group during the follow up periods.

Regarding the control (non-laser mediated osteotomy), statistical analysis of bone height using ANOVA test for repeated measures revealed significant difference in peri-implant crestal bone level changes $(\mathrm{mm})$ during the follow up intervals $(\mathrm{P} \leq 0.05)$. Simple main effect with Bonferrioni correction revealed significant difference between peri-implant crestal bone level changes $(\mathrm{mm})$ from loading - six months, 12 months and 18 months and significance difference between bone height at the interval 6-12 months. However minimal insignificant $(P \geq 0.05)$ change in the measured peri-implant crestal bone level changes $(\mathrm{mm})$ was detected at the interval 12-18 months.

Although the data obtained from table 1 revealed greater amount for the measured peri-implant crestal bone level changes $(\mathrm{mm})$ for the control group (non laser mediated osteotomy) compared to the studied group during the follow up period, statistical analysis of the data using ANOVA test for repeated measures revealed no significance difference $(P \geq 0.05)$.

\section{DISCUSSION}

Peri-implant bone loss in screw-retained restorations is a highly complex phenomenon with numerous etiologies currently mentioned in the dental literature. Osseointegrated implants supporting screw retained fixed prosthesis are subjected to both dynamic and static loading. Dynamic forces on the implants may result from chewing and can reach various magnitudes. On the other hand static loading may be produced by the tension in locking screws, when securing a misfitting framework to the implants. ${ }^{(40)}$ 
Although, natural dentition causes occlusal forces that are 5-6 times more on the single denture than complete dentures, the results of this study showed that peri-implant crestal bone level changes in Er,Cr:YSGG LASER biomodulated and non-laser mediated osteotomy in lower single denture rehabilitated with fixed detachable prosthesis constructed from BioHPP (High Performance Polymer) are within the accepted standards stated in the literature. ${ }^{(4)}$ The findings of the present study demonstrates that the two studied modalities (treatment options) individually showed significant bone loss around implants $(P<0.05)$ during the eighteen months follow up period, while insignificant difference $(P>0.05)$ was observed when comparing the peri-implant bone loss between the two groups.

Patients in this study were accurately selected and thoroughly examined to meet certain criteria as an attempt to decrease human variables and exclude any factor or habit that might adversely affect the results of this study. Split mouth design was used in this study in the lower arch where the side was randomly assigned to LASER biomodulation to eliminate a lot of inter-subject variability from the treatment effect. ${ }^{(42)}$

Bending moments developing from non-axial over loading of the implants may cause stress concentrations exceeding the physiologic adaptive capacity of cortical bone, leading to crestal bone loss and implant failures. ${ }^{(43)}$ Therefore, occlusal adjustment of the natural teeth was performed to eliminate premature and deflective occlusal contacts in centric and eccentric jaw position.

Stresses transmitted to the implant supporting fixed/detachable prosthesis are affected by the antero-posterior spread (A-P spread) of the implants and material used for its fabrication. In this study patients were rehabilitated by fixed detachable lower single denture supported by six dental implants, four implants in the interforaminal region and two implants placed in the region of the 1st molar) constructed from BioHPP (High Performance Polymer). The favorable results of this study may be attributed to the use widely distributed implants under the prosthesis, which allows for reduction of the cantilever length, increases the anteriorposterior spread, and reduces peri-implant bone stress. The favorable marginal bone resorption with PEEK may also be related to the reduced occlusal forces transmitted indirectly to the implants through PEEK. PEEK has reduced modulus of elasticity, light weight, dampening of the occlusal forces, and shock absorption capability. ${ }^{(21-24)}$

The concept of clinically acceptable fit using CAD/CAM technology encompasses the goal of reducing both biologic and mechanical complications. The application of 3-dimensional (3-D) imaging (computerized tomography scan), 3-D software for treatment planning, fabrication of computer-generated surgical guides using additive prototyping, besides CAD/CAM milling procedures significantly improves implant therapy. ${ }^{(44)}$ Clinically acceptable fit in combination with control over the occlusal forces are important for ensuring success of the implants and the restoration, this may account for the results of this study.

Comparing the amount of peri-implant crestal bone height changes between two groups, the Er,Cr:YSGG LASER biomodulated group showed lower value of crestal bone loss. Erbium LASER accelerated and improved the bone tissue healing process by detoxifying the osteotomy site before implant insertion, to remove the smear or char layer and provide a favorable surface for cell attachment and thus accelerate healing and bone formation and can explain the results of the study. ${ }^{(45)}$

In addition, the potential photo-biomodulatory effects of low powers of Erbium LASER irradiation on osteoblasts promote new bone formation by inducing proliferations and differentiation of osteoblasts.ErbiumLASER in low power parameters induce the phosphorylation of extracellular signal regulated protein kinase which is activated in increased ectoblastic proliferation to promote bone healing and adhesion to the dental implants thus improving the oesseointegration process. ${ }^{(35-38)}$ 


\section{CONCLUSION}

Within the limitation of this study findings, it seems reasonable to suggest that primary implant stability, good oral hygiene, control of occlusal load, patient compliance and passive fit are important factors for the long term success of fixed detachable lower single denture designed by CAD/CAM rather than the additional use of low level laser therapy.

\section{REFRENCE}

1. Misch C.E.: Contemporary Implant Dentistry, 3rd ed. Mosby Elsevier, St. Louis, Missouri 2008.

2. Ohkubo C., Baek K.W.: Does the presence of antagonist remaining teeth affect implant overdenture success. A systematic review. J Oral Rehab. 2010, 37: 306-312.

3. Winkler S.: Essentials of Complete Denture Prosthodontics, $2^{\text {nd }}$ ed. Philadelphia, London and Toronto W.B. Saunders Co., 1988

4. Zarb G.A., Bolender C.L., Carlsson G.E.: Boucher's Prosthodontic Treatment For Edentulous Patients. $11^{\text {th }}$ ed., St. Louis. C.V. Mosby co., 1997.

5. Ellinger C.W., Rayson J.H., Henderson D.: Single complete dentures. J Prosthet Dent.1971, 26:32-39.

6. Malo’' P., Rangert B., Nobre M.: All-on-Four immediatefunction concept with Branemark system implants for completely edentulous mandibles: A retrospective clinical study. Clin Implant Dent Relat Res, 2003, 5:2-9.

7. Malo' P., Rangert B., Nobre M.: All on 4 immediate function concept with Branemark system implants for completely edentulous maxillae: a 1-year retrospective clinical study. Clin Implant Dent Relat Res, 2005, 1:88-94.

8. Meijer H.J., Raghoebar G.M., Van 't Hof M.A.: Comparison of implant-retained mandibular over-dentures and conventional complete dentures: a 10-year prospective study of clinical aspects and patient satisfaction. Int J Oral Maxillofac Implants. 2003;18:879-885.

9. Ekelund J.A., Lindquist L.W., Carlsson G.E., Jemt T.: Implant treatment in the edentulous mandible: A prospective study on Brånemark system implants over more than 20 years. Int J Prosthodont. 2003; 16:602-608.

10. Norton M.R., Ferber C.: The nonresilient hybrid removable prosthesis: Treatment of choice for the atrophic maxilla. Int J Periodontics Restorative Dent 1999;19(2):189-197.
11. Stellingsma K., Slagter A.P., Stegenga B., Raghoebar G.M., Meijer H.J.A.: Masticatory function in patients with an extremely resorbed mandible restored with mandibular implant-retained overdentures: comparison of three types of treatment protocols. J Oral Rehabil. 2005; 32:403-441

12. Naert I., Quirynen M., van Steenberghe D., Darius P.: A study of 589 consecutive implants supporting complete fixed prostheses. Part II: Prosthetic aspects. J Prosthet Dent. 1992; 68:949-956.

13. Ferrigno N., Laureti M., Fanali S., Grippaudo G.: A longterm follow-up study of non-submerged ITI implants in the treatment of totally edentulous jaws. Part I: Ten-year life table analysis of a prospective multicenter study with 1286 implants. Clin Oral Implants Res. 2002; 13:260-273.

14. Ramasamy M., Giri N., Raja R., Subramonian A., Karthik L., Narendrakumar R.: Implant surgical guides: From the past to the present. J. Pharm. Bioallied Sci. 2013, 5:98-102.

15. White S.N.: Effect of cantilever length on stress transfer by implant-supported prosthesis. J. Prosthet. Dent.1994, 7:493-499.

16. Duyck J., Van Oostcrwyck H., Vander Sloten J. Magnitude and distribution of occlusal forces on oral implants supporting fixed prosthesis - An in vivo study. Clin. Oral implants Res. 2000, 111: 465-475.

17. Attard N.J., Zarb G.A.: Long-term treatment outcomes in edentulous patients with implant-fixed prostheses: the Toronto study. Int J Prosthodont. 2004;17: 417-424.

18. Nabhan M.S.: Effect of different fixed detachable implant supported prosthesis material on the stresses induced on the supporting structures. Egypt Dent J. 2019: 65, 445-452.

19. Shawky A.O., Lotfy S.: Zirconia versus Biohpp frameworks on stresses induced in implants supporting structures using ALL-ON- $4{ }^{\circledR}$ Protocol ( stain gauge analysis). Egypt Dent J. 2018: 64, 373-381

20. Hassel A.J., Shahin R., Kreuter A., Rammelsberg P.: Rehabilitation of an edentulous mandible with an implantsupported fixed prosthesis using an all-ceramic framework: a case report. Quintessence Int 2008; 39: 421-426

21. Zoidis P., Papathanasiou I., Polyzois G.: The use of a modified polyether-ether-ketone (PEEK) as an alternative framework material for removable dental prostheses. A clinical report. J Prosthodont 2016; 25: 580-584.

22. Skirbutis G., Dzingutė A., Masiliūnaitė V., Šulcaitė G., Žilinskas J.: A review of PEEK polymer's properties and its use in Prosthodontics. Stomatologija, Baltic Dental and Maxillofacial Journal, 2017, 19:19-23. 
23. Rosentritt M., Schneider-Feyrer S., Behr M., Preis V.: In vitro shock absorption tests on implant-supported crowns: Influence of crown materials and luting agents. Int J Oral Maxillofac Implants 2018; 33:116-122.

24. Zoidis P.: The All-on-4 modified polyetheretherketone treatment approach: A clinical report. J Prosthet Dent 2018; 119: 516-521.

25. Suwannaroop P., Chaijareenont P., Koottathape N., Takahashi H., Arksornnukit M.: In vitro wear resistance, hardness and elastic modulus of artificial denture teeth. Dent Mater J. 2011; 30: 461-468.

26. Parker S.: LASER-tissue interaction. Br. Dent. J. 2007, 202: 73-81.

27. Parker S.: Low-level LASER use in dentistry. Br. Dent. J. 2007, 202:131-138.

28. Coluzzi D.J.: Fundamentals of dental LASERs: Science and instruments. Dent. Clin. North Am. 2004, 48:751-770.

29. Walsh L.J.: The use of LASERs in implantology: An overview. J. Oral Implantol. 1992, 18:335-340.

30. Bhat M.A.: LASERs in prosthodontics- An overview Part 1: Fundamentals of dental LASERs. J. Indian Prosthodont. Soc. 2010, 10:13-26.

31. Ismaeel S.A., Abbas A.H.: The effect of low level laser on oesseointegration of dental implants. J Bagh College Dent. 2011, 23(3):112-116.

32. Walsh L.: The current status of LASER applications in implant dentistry. Aust. Dent. J. 2003. 48:146-155.

33. Lee S.Y., Piao C., Heo S.J., Koak J.Y., Lee J.H., Kim T.H., Kim M.J., Kwon H.B., Kim S.K.: A comparison of bone bed preparation with LASER and conventional drill on the relationship between implant stability quotient (ISQ) values and implant insertion variables. J. Adv. Prosthodont. 2010, 2:148-153.

34. Kusek E.R.: The use of LASER technology (Er;Cr:YSGG) and stereolithography to aid in the placement of a subperiosteal implant: case study. J. Oral Implantol. 2009, 35:5-11.

35. Zhang X., Xie S., Zhan Z., Zhao H., Guo J.: Evaluation of Er,Cr:YSGG LASER for hard tissue ablation: An in vitro study. Front. Optoelectron. China. 2010, 3:163-168.

36. Yazici A.R., Yildirim Z., Antonson S.A., Kilinc E., Koch D., Antonson D.E.: Comparison of the Er,Cr:YSGG LASER with a chemical vapour deposition bur and conventional techniques for cavity preparation: A microleakage study. LASERs Med. Sci., 2012, 27:23-29.

37. Lewandrowski K.U., Lorente C., Schomacker K.T., Flotte T.J., Wilkes J.W., Deutsch T.F.: Use of the Er, Cr:YSGG LASER for improved plating in maxillofacial surgery: Comparison of bone healing in LASER and drill osteotomies. LASERs Med. Surg.1996, 19:40-45.

38. Wang X., Ishizaki N.T., Suzuki N., Kimura Y., Matsumoto $\mathrm{K}$.: Morphological changes of bovine mandibular bone irradiated by Er,Cr:YSGG LASER: an in vitro study. LASERs Med. Surg. 2002, 20:245-250.

39. Frederiksen N.L.: Diagnostic imaging in dental implantology. Oral Surg Oral Med Oral Pathol Oral Radiol Endod. 1995, 80: 540-554.

40. Maló P., de Araújo Nobre M., Moura Guedes C., Almeida R., Silva A., Sereno N., Legatheaux J.: Short-term report of an ongoing prospective cohort study evaluating the outcomeof full-arch implant-supported fixed hybrid polyetheretherketone- acrylic resin prostheses and the All-on-Four concept. Clin Implant Dent Relat Res. 2018; 20:692-702.

41. Albrektsson T., Zarb G.A.: Determinants of correct clinical reporting. Int J. Prosthodont. 1998; 11:517-521.

42. Zhu H., Zhang S., Ahr C.: Sample size consideration for split mouth design. Stat Method Med Res.2019, 28:3487-3586

43. Akça K., Iplikçioğlu H.: Finite element stress analysis of the influence of staggered versus straight placement of dental implants. Int J Oral Maxillofac Implants. 2001; 16:722-730.

44. Takahashi T., Gunne, J.: Fit of implant frameworks: an in vitro comparison between two fabrication techniques. J Prosthet Dent. 2003, 89(3):256-260.

45. Kusek E.R.: The Er,Cr:YSGG LASER: A Perfect Fit with Implant Dentistry, J. LASER Den. 2010,18:132-134. 